Corps et culture

Numéro 6/7 | 2004

Métissages

\title{
Pascal DURET, Sociologie du sport
}

Paris, Armand Colin, Collection « Dynamiques », 2001, 198 p.

Jérôme Pruneau

\section{(2) OpenEdition}

1 Journals

Édition électronique

URL : http://journals.openedition.org/corpsetculture/980

DOI : 10.4000/corpsetculture.980

ISSN : $1777-5337$

Éditeur

Association Corps et Culture

Édition imprimée

Date de publication : 1 janvier 2004

ISSN : 1268-5631

Référence électronique

Jérôme Pruneau, «Pascal Duret, Sociologie du sport », Corps et culture [En ligne], Numéro 6/7 | 2004, mis en ligne le 11 octobre 2007, consulté le 23 septembre 2020. URL : http://journals.openedition.org/ corpsetculture/980; DOI : https://doi.org/10.4000/corpsetculture.980

Ce document a été généré automatiquement le 23 septembre 2020.

(c) tous droits réservés 


\title{
Pascal DURET, Sociologie du sport
}

\author{
Paris, Armand Colin, Collection « Dynamiques », 2001, 198 p.
}

\author{
Jérôme Pruneau
}

1 Il est louable de réaliser à l'aube du Xxi ${ }^{e}$ siècle naissant, le constat d'une sociologie du sport déjà fournie, notamment par les nombreux ouvrages qui s'attachent, depuis une trentaine d'années, à éclairer les activités physiques et sportives dans leur dimension sociale. C'est à cette tâche que s'attelle Pascal Duret dans ce livre qui, s'il n'a pas la prétention de révolutionner la compréhension sociologique des pratiques, a le mérite d'établir un bilan remarquablement illustré et de proposer une nouvelle base de travail pour les recherches à venir. Faire le point sur ce qui est en cours, sur les courants « en phase » ou "à la mode ", sur les objets " en vogue », sur les disciplines phares ou sur les méthodologies employées, constitue l'essentiel de ce volume, selon une approche de la lecture facilitée par des encadrés méthodologiques liés à l'argumentation.

2 La préoccupation première de l'auteur est de comparer les différents résultats des enquêtes réalisées sur les pratiques sportives et menées quantitativement sur le sol national depuis 1967 (la dernière étude faisant date étant celle de Mignon et Truchot, 2001). Selon une grille d'analyse qui recoupe les questions majeures liées à ces enquêtes (nombre de pratiquants, différenciation sexuelle, classe sociale, etc.), un bilan est dressé sur l'ingérence grandissante du sport dans les strates du social. Augmentation des pratiquants, certes, mais aussi distribution sexuelle affirmée selon les modalités de pratiques, les espaces et les disciplines. A ce titre les tableaux proposés servent admirablement la lecture (p. 22, 23, 24, 25).

3 Par ailleurs, l'accent est mis sur le rôle désormais déterminant du « hors club », tout en précisant, à juste titre, que celui-ci n'est pas à la hauteur des estimations " prophétisées » au milieu des années 1990, et que les pratiques sportives dites « fun » sont désormais elles aussi institutionnalisées sous des formes et à des degrés divers.

Pour ce qui est de la répartition par le social, le constat est à l'égal de la critique émise : l'extrême diffusion des pratiques semble gommer le poids des déterminations sociales. Ce qui fait dire à l'auteur « qu'il est peut être vain et inutile [...] de vouloir s'acharner à faire perdurer coûte que coûte un "système des sports" positionnant une grande quantité de pratiques qui seraient l'apanage de tels ou tels groupes sociaux » (p. 29). 
5 D'ailleurs, suite à cette donne quantitative et comparative, l'auteur glisse sur la nécessité d'une compréhension sociologique complémentaire par le biais des études qualitatives, à même de déceler les significations symboliques enfouies et difficilement accessibles à l'appareil quantitatif. L'exemple de la marche à travers les travaux de Rauch (1997) en propose une lecture, tout comme les travaux de Yonnet (1998) montrent que le système des sports ne peut se réduire à l'expression en constellations (analyse des correspondances), longuement privilégiées par les sociologues en STAPS. $\mathrm{Au}$ final de ce chapitre d'ouverture, c'est l'alternative (tout comme la complémentarité) qui est proposée par le truchement des approches qualitatives développées notamment dans le deuxième chapitre.

Ce dernier dresse de façon systématique un catalogue des enquêtes ethnologiques menées sur les pratiques sportives en démontrant l'intérêt croissant et dialectique de cette discipline vis-à-vis du champ. Sous l'impulsion de C. Bromberger (le match de football, 1995), les études ont fleuri la dernière décennie (A. Saouter, L. Wacquant, E. De Léseleuc, M. Segalen, M. Barthélemy, A. N. Waser) et ont mis en avant la dimension de l'observation, de la description et des entretiens dans la compréhension sociale des terrains d'enquête contemporains. A cet égard, un récapitulatif méthodologique profite au lecteur novice, et rappelle combien cette science sociale de la « curiosité » s'applique efficacement aux pratiques sportives par le biais de l'observation participante, celle pour qui « il faut être avec ou même faire avec» (p. 62).

7 Les quatre chapitres suivants $(4,5,6,7)$ fondent une sociologie des objets " de mode » qui se situent au carrefour des analyses et des méthodes actuelles. Violence et intégration raciale (ou insertion), éthique et dopage, médiatisation ou expression du corps sont autant de faits sociaux qui préoccupent et caractérisent pleinement l'intrication réciproque de l'objet sportif au social. Ici, P. Duret propose une contextualisation des thèmes abordés avec les études récentes ou en cours. A travers le prisme d'un sport « au carrefour » des enjeux de société, l'analyse tente de faire surgir les questions de fond qui canalisent les attentions. Sans entrer directement dans ces chapitres pour laisser la place aux lecteurs de découvrir par eux-mêmes leurs multiples intérêts, on peut noter, par exemple, sur le chapitre qui a trait à la violence, comment l'auteur tente, non sans difficultés, d'articuler les aspects empiriques relevés par les chercheurs dans leurs travaux (Duret et Augustini, 1994 ; Bui-truong, 1995 ; Chantelat, Fodimbi et Camy, 1996; Chateaureynaud et Theiller, 1998; Travert, 1997; 1999; Charrier, 1998) avec les théories de socialisation ou les approches interactionnistes. Au final, si des questions demeurent, l'intérêt réside dans le fait de les avoir dépassées en ayant pris conscience des pierres angulaires qui structurent le phénomène social (ici la violence et l'intégration) et son articulation multi-référentielle avec le sport.

8 La conclusion qui peut être portée à cet ouvrage serait de lui reconnaître toutes les qualités d'une œuvre riche dans la volonté de comprendre le sport et ses objets à travers la multitude des études réalisées ces cinq dernières années. Indéniablement, le constat est dressé, et le bilan éloquent : le sport se porte bien, la recherche sociologique qui lui est dévolue garde une vitalité à l'égal de son aura sociale. Il n'est plus un objet homogène comme on a pu l'appréhender jadis au commencement d'une sociologie de fond. Il est à percevoir dans les multiples interstices de sa complexité, entre les lignes d'une hétérogénéité qui s'écrit sous toutes les formes du social, là où il s'immisce. Par une approche qualitative complémentaire aux outils d'analyse davantage axés sur le quantitatif, les grands thèmes sociaux qui se conjuguent avec le sport sont ici 
décortiqués à leur juste mesure, même si des critiques peuvent être émises. Croiser des objets, des disciplines et des méthodes est un exercice périlleux et facilement critiquable, notamment lorsqu'il s'agit de faire le tour des études réalisées par d'autres. Pour autant, la tâche est bien accomplie car elle prend en compte les aspects méthodologiques des auteurs de référence sans jamais (ré)interpréter les faits autrement que ce qui est proposé à l'origine. Si l'on sent parfois une volonté de l'auteur de tout prendre en considération en parcourant certaines études de façon survolée, c'est davantage dans un souci exhaustif que par malversation scientifique. Cela donne la mesure d'une science sociale du sport pourvue, dont l'approche qualitative se positionne de plus en plus en élément incontournable d'une compréhension fine des phénomènes. En mêlant les sociologies au niveau des approches (comprenez ici l'ethnologie et l'anthropologie confondues) et des méthodes (quantitatives et qualitatives), la recherche en science sociale du sport sert une interdisciplinarité nécessaire dont P. Duret fait écho dans sa conclusion. On ne peut concevoir ni un seul versant des sciences du sport (sciences de la vie), ni une seule approche compréhensive pour éclairer cet objet commun qui nous anime tous, tout comme on ne peut concevoir une seule méthode d'évaluation des compétences de recherche. A cela, le livre de P. Duret donne le ton et la juste mesure de ce qu'il faut nécessairement recadrer au plus vite afin de ne pas sombrer dans un dogmatisme ravageur et stérile pour le champ. 